JÜRGEN MATZAT*

\title{
ZUM STAND DER SELBSTHILFE IN DEUTSCHLAND - UNTER BESONDERER BERÜCKSICHTIGUNG DER SUCHT-SELBSTHILFE ${ }^{* *}$
}

(Erhalten: 25. April 2008; angenommen: 19. November 2008)

In diesem Beitrag wird die in Europa einmalige Situation der Selbsthilfe in Deutschland dargestellt. Dabei geht es um deren Verbreitung, deren Arbeitsweise und deren politische Einflussnahme. Verschiedene Typen von Selbsthilfegruppen werden unterschieden (12-Schritte-Gruppen, psychologisch-therapeutische Gesprächs-Selbsthilfegruppen und Selbsthilfe-Organisationen von chronisch kranken und behinderten Menschen). Die jeweilige Arbeitsweise dieser Zusammenschlüsse wird dargestellt. Besondere Berücksichtigung findet die Sucht-Selbsthilfe, die sich in Deutschland nur teilweise auf die Anonymen Alkoholiker stützt, darüber hinaus auf eine Vielzahl anderer Selbsthilfegruppen mit dem Ziel der Abstinenz ihrer Mitglieder, vielfach im Umfeld kirchlicher Wohlfahrtsverbände. Die Besonderheit der Sucht-Selbsthilfe, die - anders als bei körperlichen Erkrankungen - ein möglicher Weg zur Genesung sein kann, wird betont. Der Beitrag endet mit Überlegungen zu möglichen Ursachen der Selbsthilfe-Bewegung, die sich vor allen Dingen in den hochindustrialisierten Staaten Mittel-, West- und Nordeuropas in den letzten zwei oder drei Jahrzehnten entwickelt hat. Einige Meilensteine, die den deutschen Weg markieren, werden geschildert: die Rolle der Forschung, die Unterstützung durch staatliche Gesetzgebung und schließlich die Verpflichtung der gesetzlichen Krankenversicherung zur finanziellen Förderung der Selbsthilfe.

Schlüsselbegriffe: Selbsthilfegruppe, Selbsthilfe-Organisation, Selbsthilfe-Kontaktstelle, SuchtSelbsthilfe, Laienhilfe, ehrenamtliches/bürgerschaftliches Engagement, Forschung, Gesetzgebung

\footnotetext{
* Jürgen Matzat, Kontaktstelle für Selbsthilfegruppen der Deutschen Arbeitsgemeinschaft Selbsthilfegruppen e. V., Friedrichstr. 33, D-35392 Gießen, Deutschland; Juergen.Matzat@psycho.med.unigiessen.de

${ }^{* *}$ Diesen Text widme ich Prof. Wolfgang Slesina, Universität Halle, der sich durch seine Forschung auf dem Gebiet der Selbsthilfegruppen und insbesondere der Kooperation von Ärzten und Selbsthilfegruppen große Verdienste um die Selbsthilfebewegung in Deutschland erworben hat.
}

1788-4934 / \$20.00 (C) 2009 Akadémiai Kiadó, Budapest 
The Present State of Self-Help in Germany - with Special Regard to Addiction Self-Help: This article presents the self-help scenery in Germany, which is unique in Europe. It deals with the dissemination of self-help groups and organizations, their various ways of proceedings, and their political influence. Different types of self-help groupings are described (12-steps-groups, psychologico-therapeutic talking-groups, and self-help organizations of chronically ill and handicapped persons). Special attention is given to self-help groups of addicted persons. In Germany only a small part of them are Alcoholics Anonymous. Beyond that a variety of other selfhelp groups devoted to abstinence of their members exist, in many cases attached to welfare organizations of the Catholic or Protestant Church. A specific factor in self-help groups for addicted persons is the possible recovery from the disease, which is not the case in self-help groups of chronically ill and handicapped persons. Finally the article presents some ideas about possible causes for the self-help movement, which developed over the last two or three decades, particularly in highly industrialized countries of Central, Western and Northern Europe. Some milestones along the German way to self-help are mentioned: the important role of research(ers), the support by state's legislation, and finally the obligation of Germany's statutory health insurance to support self-help financially.

Keywords: self-help group, self-help organization, self-help clearinghouse, addiction, volunteerism, research, legislation

Die Selbsthilfebewegung hat in Deutschland ein Ausmaß erreicht wie in keinem anderen europäischen Land. Bei einer Bevölkerung von ca. 82 Millionen wird die Zahl der örtlichen Selbsthilfegruppen auf bald 100.000 geschätzt, die der in Dachverbänden zusammengeschlossenen Selbsthilfeorganisationen auf über 100, die der bereichsübergreifenden Selbsthilfe-Kontaktstellen vor Ort auf etwa 300. Die Selbsthilfe-Landschaft hier ist gekennzeichnet von äußerster Buntheit und Vielfalt (MATZAT 2004). Die Gruppen und Organisationen unterscheiden sich nach Größe, Struktur, Arbeitsweise, Zielsetzung, Alter und Dauerhaftigkeit, Grad der Professionalisierung und vor allem natürlich nach den Themen, die dort bearbeitet werden sollen. Meist ist es eine chronische Erkrankung, eine Behinderung, eine Sucht oder eine besonders belastende Lebenssituation. Etwa 75\% aller Selbsthilfegruppen sind dem Gesundheitsbereich zuzuordnen, auf den sich dieser Artikel im Wesentlichen bezieht. Es lassen sich vor allem drei Stränge der Selbsthilfe-Entwicklung identifizieren und als grobe Typologie zur Orientierung nutzen.

\section{Formen kollektiver Selbsthilfe}

\subsection{Anonymous-Gruppen}

Alle Selbsthilfegruppen des Anonymous-Typs lassen sich zurückführen auf die Anonymen Alkoholiker, die wohl berühmteste Selbsthilfegruppe der Welt. Diese überliefern einen regelrechten „Schöpfungsmythos“ von Bob und Bill, scheinbar zwei hoffnungslosen Säufern, die im Jahre 1935 in Akron/Ohio (USA) herausfanden, dass sie nicht zu trinken brauchten, solange sie im Gespräch miteinander waren, und dass sie sich als gleichermaßen Betroffene besser verstehen und unterstützen konnten, als es 
professionelle Therapeuten während ihrer erfolglosen Entziehungskuren je vermocht hatten. Daraus entwickelte sich eine weltweite Bewegung, der sich nach und nach auch Angehörige von Alkoholkranken, andere Drogenabhängige, Spielsüchtige, Menschen mit Essstörungen und seelischen (,emotionalen“) Problemen, neuerdings sogar Kauf- und Liebessüchtige angeschlossen haben. In Amerika wird dieses spezielle Konzept mitunter sogar mit Selbsthilfegruppen insgesamt gleichgesetzt. All diese Gruppen folgen einem Programm der „12 Schritte“, sie akzeptieren eine „höhere Macht“" (so wie jeder sie persönlich versteht) und arbeiten nach einem ganz bestimmten Konzept. Sie sind stets „offen“, d.h., jederzeit können neue Interessierte hinzustoßen, wenn sie nur den Wunsch haben, zu genesen und „trocken“ zu werden. Die Gruppengespräche bestehen im Wesentlichen aus einer Reihe von Monologen, in denen aus dem eigenen Leben, von der eigenen Sucht, von den Anstrengungen, sie zu überwinden, von Rückfällen und von Erfolgen berichtet wird. Dabei soll jeder von sich selbst sprechen; Fragen oder gar Ratschläge an andere sind verpönt.

Die Balance zwischen Selbst- und Fremdhilfe ist für die Philosophie der Anonymous-Gruppen von zentraler Bedeutung. Für jeden in der Runde ist völlig klar, dass er oder sie um seiner/ihrer selbst willen, zur Überwindung der eigenen Krankheit in die Gruppe geht und nicht, um anderen dort zu helfen. Zugleich ist jeder verpflichtet, zum Erhalt der Gemeinschaft beizutragen und die eigenen Erfahrungen weiterzugeben. Selbsthilfe und Engagement für andere sind untrennbar miteinander verbunden. Beides geschieht im selben Moment und am selben Ort, nämlich im „Meeting“, wie die Treffen genannt werden. Was zählt, ist die Beteiligung hier. Eine andere Form von Mitgliedschaft gibt es gar nicht und ehrenamtliches Helfertum nur in sehr geringem Maße, etwa bei den sog. „Sponsoren“ - das sind erfahrene Mitglieder, die eine Art Patenschaft für Neue übernehmen - oder bei den „Diensten“ für die Gemeinschaft.

\subsection{Psychologisch-therapeutisch orientierte Gesprächs-Selbsthilfegruppen}

Ohne ein festes Programm, wie es bei allen Anonymous-Gruppen vorherrscht, aber ebenfalls mit im weitesten Sinne psychotherapeutischer Zielsetzung haben sich Selbsthilfegruppen diesen Typs in Deutschland seit den späten 70er Jahren in großer Zahl gebildet (MOELLER 1996, 2007). Im Jahre 2003 ergab die Recherche im Rahmen eines eigenen Forschungsprojekts (HÖFLICH et al. 2007) ca. 5.000 solcher „Psycho-Selbsthilfegruppen" in Deutschland. In der Regel sind es örtliche Gesprächskreise ohne Anschluss an größere Verbände auf Landes- oder Bundesebene. Etwa fünf bis zehn Menschen finden sich auf Initiative eines Betroffenen zusammen, oft mit Unterstützung durch Fachleute z.B. in Selbsthilfe-Kontaktstellen (HERZOG-DiEM \& HUBER 2007), um im gemeinsamen Gespräch ihren Problemen auf den Grund zu gehen und zu Lösungen zu finden. Sie selbst definieren den Grund ihres Zusammenkommens und das Thema ihrer Gruppenarbeit, sei es durch psychodiagnostische Fachbegriffe (z.B. „Depression“ oder „Panik- und Angststörungen“), bestimmte Verluste (z.B. „trauernde Eltern“ oder „Geschiedene“), spezielle familiäre Belastungen (z.B. „Angehörige von psychisch Kranken“ oder „Eltern allergiekranker Kinder“), traumatische Erfahrungen 
(z.B. „,sexueller Missbrauch“ oder „erwachsene Kinder von Alkoholikern“) oder durch chronische Erkrankung oder Behinderung (z.B. „Insuliner“ oder „Stotterer"). Sie treffen sich ohne Anleitung durch eine Fachkraft zu regelmäßigen Gesprächen, manchmal auch zu gemeinsamen Freizeitaktivitäten. Gearbeitet wird mit denen und für jene, die anwesend sind; die Gruppe ist der zentrale Ort des Geschehens. Andere vom gleichen Schicksal Betroffene interessieren erst, wenn sie als potenzielle Gruppenmitglieder in Frage kommen.

Diese Gruppen sind zwar grundsätzlich bereit, neue Mitglieder aufzunehmen, tun dies aber vor allem mit dem Ziel, den eigenen Bestand zu erhalten, und nicht, um andere zu versorgen. Zeitweilig können sie sogar ganz geschlossen bleiben, um sich innerlich zu stabilisieren und ihre Arbeitsfähigkeit zu gewährleisten. Wer beitritt, soll nach einer gewissen Eingewöhnungszeit verlässlich entscheiden, ob er oder sie auf Dauer bleiben und dann auch zum Erhalt der Gruppe und zu einem produktiven Gruppenprozess beitragen will. Das bedeutet vor allem die Bereitschaft, sich zu öffnen, über persönliche Belange, Gefühle und Ansichten zu sprechen, eigene Erfahrungen mitzuteilen und aus denen anderer zu lernen. Es geht darum, Beziehungen zu den übrigen Mitgliedern aufzunehmen und an deren Schicksal teilzuhaben, sich in deren Problematik einzufühlen, ohne vorschnell mit einem Ratschlag zur Hand zu sein, für sich selbst und für die Selbsthilfegruppe als Ganzes Verantwortung zu übernehmen. Dies alles zu tun, und zwar ohne professionelle Anleitung, vielfach mit geringer oder gar keiner Gruppenerfahrung, weitgehend im Vertrauen auf die eigenen Kräfte und auf die der Mitbetroffenen, das verlangt schon ziemlich viel von einem Menschen: nicht nur einen offen eingestandenen Leidensdruck, sondern auch Mut und Engagement, Experimentierfreude und Bereitschaft, sich auf Neues einzulassen, sowie soziale Kompetenz und die Fähigkeit, sich in eigene und fremde seelische Prozesse einzufühlen (HÖFLICH et al. 2007). In solchen notwendigen Voraussetzungen (,Ressourcen“) liegt auch der Hauptgrund dafür, dass Selbsthilfegruppen diesen Typs für viele Menschen eben kein angemessenes Instrument sind und weshalb viele sie auch schnell wieder verlassen. Wer es jedoch schafft, auf Dauer in einer solchen Gesprächsgemeinschaft mitzuarbeiten, der kann Erfolge erzielen, die denen einer professionellen Gruppentherapie durchaus vergleichbar sind (MATZAT in press-b).

\subsection{Selbsthilfe-Organisationen}

Der dritte Strang sind die so genannten Selbsthilfe-Organisationen, die sich in unserem Land vor allem in den 70er Jahren gebildet haben - wenn auch einige Klassiker wie die Lebenshilfe für geistig Behinderte oder der Deutsche Blindenverband noch um etliches älter sind. Der Deutsche Allergie- und Asthmabund, gegründet 1897 als Heufieber-Bund, konnte sogar schon seinen 100. Geburtstag feiern. Auch die älteren Verbände der Abstinenzbewegung wie Blaues Kreuz, Guttempler oder Kreuzbund stammen bereits aus dem 19. Jahrhundert, haben aber erst seit den 70er Jahren ihren Charakter als Selbsthilfeorganisation von Betroffenen stärker entwickelt. 
Die Verbände chronisch kranker und behinderter Menschen sind vielfach auf Initiative und unter Mitwirkung von Medizinern entstanden, die ihre Patienten ermutigten, ihre Interessen selber zu vertreten und öffentlich auf ihr Schicksal und auf Mängel im Versorgungssystem aufmerksam zu machen. Man organisiert sich nach den Vorgaben des deutschen Vereinsgesetzes mit Satzungen, Vorstandswahlen, Mitgliedsbeiträgen usw. und nach den föderativen Strukturen unseres Landes in Bundes- und Landesverbänden, sowie mit lokalen Selbsthilfegruppen (oder zumindest einzelnen Verbandsmitgliedern als betroffenen Kontaktpersonen vor Ort). Die Hauptziele der Aktivitäten sind vielfach die Aufklärung und Information von Patienten über diagnostische, therapeutische und rehabilitative Möglichkeiten und Rechtsansprüche sowie die „Selbsthilfe nach außen“, d.h. Einflussnahme auf Politik und Verwaltung, vor allem aber auf Versorgungsangebote, Qualifikation von Fachpersonal und Intensivierung von Forschung. Viele dieser Selbsthilfe-Organisationen sind inzwischen als Akteure in unserem stark von Interessen bestimmten Gesundheitswesen akzeptiert. Für diese Funktion als Lobby, als eine Art „Patienten-Gewerkschaft“", ist es unerlässlich, möglichst viele Mitglieder zu haben, die zu vertreten man demokratisch legitimiert ist. In der Politik wiegt das Argument der großen Zahl bekanntlich schwer, was Probleme für Vereinigungen mit sich bringt, die ein seltenes Krankheitsbild vertreten. Die Aktivität des einzelnen Mitglieds, der persönliche Beitrag (über die jährliche Zahlung des Mitgliedsbeitrags hinaus) zum Geschehen innerhalb des Verbandes oder der örtlichen Gruppe spielt dabei zunächst eine geringere Rolle. Wie bei anderen Vereinen und Großorganisationen auch, kann man durchaus in einer völlig passiven Haltung verharren, sozusagen in einer klassischen Patienten-Rolle, die Dienstleistungen des Verbandes jedoch gelegentlich dankbar in Anspruch nehmen. Man könnte dann von „Selbsthilfe-Konsumenten" sprechen.

Viele dieser Verbände haben inzwischen hauptamtliches Personal eingestellt, insbesondere um die Geschäfte ihrer Verbandszentrale zu führen, aber auch um ihre Mitglieder und andere Betroffene fachlich zu beraten, Zeitschriften herauszugeben, Tagungen und Seminare zu organisieren. Der Dienstleistungs-Gedanke tritt bei einigen immer stärker in den Vordergrund. Das verbleibende Selbsthilfe-Element besteht dann im Wesentlichen aus der „Regie durch Betroffene“ (den Vorstand, die Mitgliederversammlung), die das Personal aussuchen und allemal mehr Einfluss auf die Programmgestaltung nehmen können als bei den Angeboten anderer Träger, etwa von öffentlichen Einrichtungen oder gemeinnützigen Wohlfahrtsverbänden.

Ein Großteil der Dienstleistungen der Selbsthilfeorganisationen wird jedoch ehrenamtlich von den betroffenen Mitgliedern selbst erbracht, ohne Honorar oder allenfalls gegen geringe Entschädigung. Hier liegt ein schier unerschöpfliches Potential von Mitmenschlichkeit, Solidarität und Gemeinsinn, welches bei den derzeit stattfindenden Diskussionen um Ehrenamt, bürgerschaftliches Engagement und freiwillige Arbeit gerne übersehen wird (MATZAT in press-a).

Als neue Aufgabe wächst dieser „verfassten Selbsthilfe“ vermehrt die Rolle zu, als Repräsentant von Patienteninteressen in allen möglichen Gremien des Gesundheitswesens mitzuwirken, um einerseits die Erfahrungen der Betroffenen als Beitrag zur Qualitätssicherung bzw. -verbesserung einzubringen und andererseits ein Element von 
„Kundenorientierung“ in unser ansonsten stark anbieterorientiertes Gesundheitswesen einzuführen (vgl. auch HäRTER et al. 2005). Seit dem Jahr 2004 sitzen Betroffene aus solchen Verbänden als Patientenvertreter im sog. Gemeinsamen Bundesausschuss, dem höchsten Gremium der Selbstverwaltung im deutschen Gesundheitswesen. Gesetzliche Grundlage hierfür bildet der $\S 140 \mathrm{f}$ des $\mathrm{SGB}^{1} \mathrm{~V}$.

\section{Zur Einordnung der Sucht-Selbsthilfe}

Die Deutsche Hauptstelle für Suchtfragen (DHS) bildet das Dach für eine große Anzahl von Gruppen der Abstinenz- und Selbsthilfeverbände in der Suchtkrankenhilfe. Diese Verbände sind: Blaues Kreuz in Deutschland (BKD), Blaues Kreuz in der Evangelischen Kirche (BKE), Bundesarbeitsgemeinschaft der Freundeskreise für Suchtkrankenhilfe (BAG), Deutscher Guttempler-Orden (I.O.G.T.) und Kreuzbund. Die Anonymen Alkoholiker (AA) gehören - entsprechend ihrem Unabhängigkeitsgebot - der DHS nicht an. In Deutschland treffen sich wöchentlich etwa 200.000 Menschen in ca. 7.500 Sucht-Selbsthilfegruppen. Nach Thematik (Art des Krankheitsbildes), weltanschaulichem Hintergrund (meist explizit christlich-humanistischer Art) und Grad der Integration in das reguläre Versorgungssystem (hohe Akzeptanz bei vielen Profis, auch nach eigenem Verständnis ein „Element im Behandlungsverbund“) sind in der Sucht-Selbsthilfe Besonderheiten festzustellen, die sie von anderen Selbsthilfebereichen unterscheidet.

Süchte, namentlich der Alkoholismus, der in Deutschland erst 1968 per Gerichtsbeschluss zu einer behandlungsbedürftigen Krankheit erklärt wurde, unterscheiden sich ihrem Wesen nach von chronischen organischen Erkrankungen wie Diabetes, Rheuma oder Neurodermitis. So spielen seelische und soziale Faktoren bei Entstehung und Verlauf von Abhängigkeiten eine sehr viel größere Rolle. Und sie können in vielen Fällen ,geheilt“ oder zumindest zum Stillstand gebracht werden, falls es zu entsprechenden Verhaltensänderungen der Betroffenen (eventuell auch ihrer Angehörigen) kommt. Wenn dies gelingt, dann in der Regel nicht durch Medikamente oder Operationen, also klassisch medizinische Eingriffe, sondern durch psychosoziale Maßnahmen, vor allem therapeutische Gespräche, Gruppenarbeit und Änderung sozialer Beziehungen. Auch wurde die Bedeutung von Angehörigen, sowohl für die Krankheitsentstehung wie für die Genesung, früh erkannt, und sie wurden als Mit-Erkrankte (Co-Alkoholiker) in ihrem eigenen Leiden und Unterstützungsbedarf wahrgenommen und aufgenommen. All dies hat sich in der Art und Weise der Selbsthilfegruppen-Arbeit im Suchtbereich niedergeschlagen: Das Gruppengespräch der Betroffenen steht absolut im Vordergrund, Regelmäßigkeit und Dauerhaftigkeit der Teilnahme sind das Geheimnis des Erfolgs, ebenso Offenheit und Ehrlichkeit vor sich selber und vor den anderen, sowie das Wissen darum, dass die Besserung letztlich aus jedem einzelnen selber kommen muss (nicht von noch so kundigen Helfern), aber doch meistens nicht

\footnotetext{
${ }^{1}$ Sozialgesetzbuch der Bundesrepublik Deutschland.
} 
aus eigener Kraft geschafft werden kann. „Du allein kannst es, aber Du kannst es nicht allein!“, sagen die Anonymen Alkoholiker.

Im Unterschied zu anderen Selbsthilfegruppen, wo die medizinische Aufklärung und Information der Patienten eine große Rolle spielt, wo deswegen gerne Ärzte zu Vorträgen eingeladen werden, wo man Medikamente und Behandlungsmethoden diskutiert, wo oft große Hoffnung auf zukünftige Erfolge der Medizin gesetzt werden, die eines Tages diese Krankheit „besiegen“ möge, wo die Qualität einzelner Ärzte oder Kliniken erörtert werden, bleiben Sucht-Selbsthilfegruppen viel stärker auf sich selber konzentriert, auf ihre Mitglieder und die ihnen innewohnenden „Heilkräfte“. (In Sucht-Selbsthilfegruppen arbeitet ein erheblicher Teil der Mitglieder (ca. 25\%) erfolgreich an ihrer Genesung, ohne jemals professionelle Hilfe in Anspruch genommen zu haben - in organmedizinischen Selbsthilfegruppen ist das praktisch undenkbar.) Insofern hat es auch keinen Sinn, bei einer Sucht-Selbsthilfegruppe lediglich anzurufen oder „mal eben vorbeizugehen“, um Broschüren mit Ernährungstipps, Hinweise auf Vor- und Nachteile bestimmter Operationsmethoden bzw. mögliche Nebenwirkungen von Medikamenten, Informationen über kommunale Fahrdienste für Behinderte oder über sozialrechtliche Regelungen zur Erlangung eines Schwerbehindertenausweises in Erfahrung zu bringen. Die Zunahme solcher „Selbsthilfe-Konsumenten“ wird von anderen Gruppierungen immer häufiger beklagt.

Aus den nicht-medizinischen Wurzeln der Sucht-Krankenhilfe stammt ein zweiter wesentlicher Unterschied zur Selbsthilfe in anderen Bereichen: die stark christlich geprägte Philosophie. In den meisten Sucht-Selbsthilfegruppen spielt die Vorstellung einer „Höheren Macht“, zumeist das christliche Gottesbild, eine große, für viele die entscheidende Rolle. Der Psychoanalytiker C. G. Jung, der in diesem Zusammenhang immer gerne als Kronzeuge zitiert wird, prägte hierfür in seinem Briefwechsel mit Bill, einem der Gründer von AA die elegante Formel ,spiritus contra spiritum“. Geistige Kräfte, so meinte er, hätten am ehesten eine Chance gegen den Weingeist. Es ist ja auch gar nicht lange her, da war die Bekämpfung des „Teufels Alkohol“ im wesentlichen eine Sache der Kirchen, und eben nicht der Medizin. Und bis heute ist das professionelle Hilfesystem im Suchtbereich nur teilweise medizinalisiert (Sucht-Klinik, Psychiatrie, Entzugsbehandlung). Der ambulante Bereich (Sucht-Beratungsstellen) gehört in Deutschland zur Sozialarbeit, oft in Trägerschaft der großen Wohlfahrtsverbände, mit mehr oder weniger christlicher Ausrichtung.

In professionellen Suchthilfe-Einrichtungen, stationär wie ambulant, arbeiten viele Menschen, die „doppelt qualifiziert“ sind, d.h. sie verfügen neben ihrer „erlernten Kompetenz" als ausgebildete Fachleute auch über „erlebte und erlittene Kompetenz“ (vgl. BORKMAN 1999) als (ehemals) Süchtige. Es ist typisch für den Suchtbereich, dass eigene Erfahrung mit einer solchen Erkrankung als eine wesentliche - manche meinen sogar, als eine hinreichende - Qualifikation für die Übernahme der Helferrolle angesehen wird. Gelegentlich glaubt man als Außenstehender sogar eine gewisse Überheblichkeit bei solchen „Berufs-Betroffenen“ zu spüren, sie wüssten es (alles?) besser als die „nur“ ausgebildeten Fachleute. Betroffenheit kann eben auch zum Mythos werden. In jener Grauzone zwischen Betroffenheit, ehemaliger Betroffenheit, ehrenamtlichem und bezahltem Helfertum bewegen sich die Verbände der Sucht- 
Selbsthilfe mit den von ihnen angebotenen Ausbildungsgängen für Laien zum ehrenamtlichen „Suchtkrankenhelfer“. Während in der ursprünglichen Idee der Selbsthilfegruppe die Gleichheit der Betroffenen und die Wechselseitigkeit des Erfahrungsaustauschs und der Unterstützung zentrale Momente sind, werden hier nun plötzlich einige „etwas gleicher“. Der Selbsthelfer wird zum Laienhelfer, zunächst in der Regel ehrenamtlich, in manchen Fällen dann sogar auch gegen Geld. Andererseits stellen diese hoch motivierten und weitergebildeten Sucht(selbst)helfer sicher ein besonders verlässliches Element der Gruppenarbeit dar, und sie wirken häufig als eine Art Botschafter oder Vorposten der Sucht-Selbsthilfe in Betrieben, Vereinen, Nachbarschaft und persönlichem Bekanntenkreis. Eine solche „Karriere“, ein solcher Rollenwechsel, ein solcher „Wechsel auf die andere Seite“ ist spezifisch für den Suchtbereich und bei anderen Krankheitsbildern in dieser Form nicht zu finden. Eine gewisse Parallele weisen allerdings manchmal die speziellen Fortbildungsangebote anderer Selbsthilfe-Organisationen für die Leiter ihrer örtlichen Gruppen auf, wo ebenfalls ein Informationsvorsprung geschaffen oder ausgebaut wird, der hierarchischen Elementen Vorrang vor egalitären einräumt. Es kommt dort jedoch nicht zu formalen Abschlüssen mit entsprechender Zertifizierung. Natürlich sind sich die Verbände der Sucht-Selbsthilfe über dieses Spannungsfeld im Klaren, und es wird in den Schulungen darüber gesprochen.

Zusammenhänge zwischen persönlicher Betroffenheit und Motiven der Berufswahl gibt es unter ausgebildeten Helfern ebenfalls, und sie sind keinesfalls ehrenrührig. Der deutsche Psychoanalytiker W. SchmidBaUER $(1977,2007)$ hat vor vielen Jahren dafür die eingängige (allerdings auch all zu leicht missverstandene und missbrauchte) Formel von den ,hilflosen Helfern“ geprägt. Unter Psychotherapeuten z.B. gilt dies längst als Binsenweisheit, und in ihrer Ausbildung werden solche Zusammenhänge thematisiert und (hoffentlich ausreichend) bearbeitet. Selbst unter den ach so objektiv-naturwissenschaftlichen Medizinern spielen persönliche oder familiäre Schicksale oft eine große Rolle bei der Berufswahl. So berichtete eine Diabetologin, unter ihren Fachkollegen gäbe es einen hohen Anteil von Diabetikern, worüber allerdings nicht offen gesprochen werde. Inzwischen gehört es in Deutschland zum Standard der Mediziner-Ausbildung, dass mit den jungen Studenten solche Fragen reflektiert werden.

Jene „offene Grenze“ zwischen Betroffenen- und Fach-Kompetenz im Suchtbereich findet ihre Entsprechung in der hochgradigen Akzeptanz der Sucht-Selbsthilfe im Suchthilfe-System insgesamt. Sie gilt allseits als notwendiges „Glied in der therapeutischen Kette“. Es ist in Deutschland praktisch nicht mehr vorstellbar, dass jemand wegen Alkoholismus in einer Klinik behandelt oder in einer Beratungsstelle betreut wird, ohne dass er auf Abstinenzgruppen hingewiesen würde. Dieses zu unterlassen, wäre geradezu ein „Kunstfehler“! Auch hier haben wir es wieder mit einem Spezifikum der Sucht-Selbsthilfe zu tun, welches sie von anderen Feldern der Selbsthilfe unterscheidet. Allerdings holen andere Indikationsbereiche mächtig auf: Inzwischen werden auch viele Diabetiker, Rheumatiker, Krebspatienten usw. von ihren Behandlern auf Selbsthilfegruppen hingewiesen. Man erhofft sich vor allem eine psychische Stabilisierung und soziale Betreuung der Patienten, um die eklatanten Mängel unseres 
Medizinsystems an dieser Stelle zu kompensieren, sowie eine bessere Krankheitsbewältigung und soziale Integration (nicht jedoch Genesung!). Der Suchtbereich hatte und hat hier eine wichtige Vorreiterfunktion für das gesamte Medizinsystem. Ganz allmählich sickert auch zu den Fachleuten in den organmedizinischen Fächern durch, über welche Quellen für Information, Unterstützung, Ermutigung und Verbesserung der Lebensqualität die Patienten selber verfügen, speziell im Kollektiv von Selbsthilfegruppe und Selbsthilfe-Organisation. Diese Ressource gilt es noch stärker zu nutzen und in guter Kooperation mit professionellen Maßnahmen zu kombinieren, zum Nutzen für die Patienten, aber auch für das Gesundheitswesen als Ganzes - einschließlich seiner Finanzierung.

\section{Ursachen der Selbsthilfe-Bewegung}

\subsection{Die Verschiebung des Krankheitsspektrums}

Durch die enormen Erfolge der High-Tech-Medizin mit modernem Rettungswesen, Intensivmedizin, Transplantationen usw. gelingt es, immer mehr Menschen auch nach schwersten Unfällen und Operationen oder bei frühen tödlichen Erkrankungen am Leben zu erhalten. Oft ist aber eine endgültige Heilung, d.h. ein dauerhaftes Verschwinden der Symptome oder Behinderungen nicht möglich. Die körperlichen Folgen können allenfalls gelindert werden, von den psychischen und sozialen ganz zu schweigen. Chronische Leiden und solche Erkrankungen, die weitgehend durch seelische Konflikte und soziale Lebensweisen bedingt sind, stellen heute die zentralen Herausforderungen unseres Medizinsystems dar, nicht mehr die akuten, „besiegbaren“ Krankheiten. Die Krankheitsverarbeitung, das Leben mit Krankheit und Behinderung auf Dauer ist eine Aufgabe, mit der immer mehr Menschen konfrontiert sind. Dafür wird die Unterstützung und die Erfahrungskompetenz von anderen Betroffenen in Selbsthilfegruppen gesucht.

\subsection{Der Zerfall traditioneller sozialer Netze}

Die Bindungen, auf die man sich früher im Falle von Krankheit oder Krise verlassen konnte, stehen heute in den modernen ,postindustriellen“ Gesellschaften nicht mehr so selbstverständlich zur Verfügung. Flexibilität gilt als höchste Tugend (vgl. SENNETT 1998). Aus Eheleuten („,bis dass der Tod euch scheidet“) sind „Lebensabschnittspartner" geworden; wo früher eine Kinderschar tobte, wächst heute ein Einzelkind auf; Nachbarschaft bedeutet wenig in Hochhaus-Ghettos und bei der erhöhten Mobilität der Bevölkerung auf ihrer Suche nach Ausbildungs- und Arbeitsplätzen; Kirchengemeinden verlieren ihre soziale Bindungskraft; herkömmliche „Milieus“ (etwa in der Tradition der Arbeiterbewegung) lösen sich unter dem Druck der Modernisierung auf; Vereine und Verbände beklagen Mitgliederschwund; Sport ist für viele nicht mehr im Verein und in Mannschaften am schönsten, sondern einsam und alleine beim Joggen 
oder im Fitness-Studio. (Der amerikanische Sozialforscher R. PUTNAM (2000) fand dafür das eindrucksvolle Bild vom Bowling Alone.) Die Beispiele ließen sich fortsetzen. Einsamkeit und Isolation sind aber von der Forschung als bedeutsame gesundheitliche Risikofaktoren identifiziert worden!

Gegen solchen Beziehungsverlust setzen Selbsthilfegruppen ein neues Netzwerk. Freilich ein künstliches, wenn man so will; eines, das sich an einem bestimmten umschriebenen Zweck festmacht, z.B. der gemeinsamen Bewältigung einer chronischen Krankheit oder einer speziellen sozialen Belastungssituation.

\subsection{Der (Selbst-)Bewusstseinswandel nach den 68er Jahren}

In verschiedenen sozialen Bewegungen (z.B. Frauen-, Friedens-, Umwelt- und Gesundheitsbewegung) hat sich die Grundhaltung ausgedrückt bzw. entwickelt, nicht mehr ausschließlich den selbst ernannten oder von Autoritäten vorgesetzten Experten zu vertrauen, sondern sich selber kundig zu machen und Verantwortung zu übernehmen. In den westeuropäischen Ländern, insbesondere in Deutschland, ist dies symbolisch verknüpft mit dem Jahr 1968, als die Studenten- und Jugendrevolte starke kulturelle Veränderungen auslöste. Die Selbsthilfe-Bewegung ist ohne diese anderen sozialen Bewegungen nicht denkbar. Und natürlich ist sie auch zu sehen als eine ganz wichtige Form bürgerschaftlichen sozialen Engagements - für sich selbst und für andere Betroffene. Das wohlverstandene Eigeninteresse und die Sorge für andere kommen hier gleichermaßen zu ihrem Recht. Diese Form von bürgerschaftlichem Engagement wird eben nicht ganz freiwillig ausgeübt, sondern aus Not und mit persönlichem "Leidensdruck“.

Für die Länder des früheren Warschauer Pakts spielen vielleicht die Jahre 1989/90 eine ähnliche Rolle. Der Fall der repressiven Regime ist eine kollektive Erfahrung der dort lebenden Menschen, die jetzt - sozusagen verspätet - eine Zivilgesellschaft entstehen lässt, wo früher selbst bestimmte Gemeinschaftsaktivitäten außerhalb staatlicher Organisation, also z. B. Selbsthilfegruppen, praktisch kaum möglich waren. Wir haben dies in Deutschland nach der Wiedervereinigung erlebt.

\subsection{Bildung und Zugang zu Informationen}

Unsere Gesellschaft ist geprägt von einer rapiden Zunahme der (formalen) Bildung, - etwa gemessen an dem Prozentsatz der Abiturienten eines Jahrgangs -, und vom wachsenden Einfluss neuer Informationstechnologien, wie etwa dem Internet. Dessen Stellenwert für den breiten Zugang zu gesundheitsbezogenen Informationen wird zwar vielfach überschätzt, dürfte sich aber in Zukunft deutlich erhöhen. Der gerade stattfindende Aufbau spezieller Patienten-Informations- und -Beratungs-Systeme wird hier ebenfalls von großer Bedeutung sein. Die Arzt-Patient-Beziehung ändert sich dadurch signifikant. Der „mündige Patient“ erscheint als ein neues Leitbild im Gesundheitswesen, mit dem Entscheidungen gemeinsam zu treffen sind (im Englischen ,shared 
decision making"), welche früher allein den Fachleuten oblagen. Nichts soll mehr ohne Zustimmung des Patienten erfolgen, und dafür muss er hinreichend informiert und aufgeklärt worden sein.

\section{Meilensteine in den letzten $30 \mathrm{Jahren}$}

\subsection{Forschung}

Bis Ende der 70er Jahre kamen Selbsthilfegruppen in der öffentlichen Diskussion in Deutschland praktisch nicht vor. Wohl gab es schon welche (etwa die Anonymen Alkoholiker und andere Abstinenzgruppen, die Lebenshilfe für das geistig behinderte Kind, die Frauenselbsthilfe nach Krebs), sie waren aber für Außenstehende kein Thema, nicht für die Wissenschaft, nicht für die Medizin, nicht für die Krankenversicherung, nicht für Politik und Verwaltung. Dies änderte sich erstmals durch Forschungsprojekte an der Psychosomatischen Universitätsklinik in Gießen (1977) und am Institut für Medizinische Soziologie der Universität Hamburg (1979). Seither haben Selbsthilfe-Forscher wie M.L. MOELLER $(1996,2007)$, A. Trojan (TROJAN \& EsTORFFKLEE 2004) und W. Slesina (SLESINA \& KNERR 2007) die Entwicklung in Deutschland wissenschaftlich begleitet und mit ihren Befunden zur Akzeptanz der Selbsthilfe in unserem Land entscheidend beigetragen. Dies ist in Europa einmalig und kann als eine Erklärung für den besonderen Erfolg der Selbsthilfe in Deutschland herangezogen werden (BORGETTO 2004).

Aus dem Forschungsprojekt in Gießen entwickelte sich eine Fachgesellschaft (keine Dachorganisation von Selbsthilfegruppen!): die Deutsche Arbeitsgemeinschaft Selbsthilfegruppen e.V. In ihr schlossen sich Fachleute, Betroffene und Sympathisanten der Selbsthilfegruppen-Idee zusammen, um diese zu fördern und zu verbreiten, unabhängig von der jeweils zu bearbeitenden Thematik. Ein „Leitmedium“ dieses Verbandes ist sein Selbsthilfegruppenjahrbuch (1999 bis 2009). Ausgehend von den hier zusammengetragenen Erfahrungen wurde das Konzept einer „Kontaktstelle für Selbsthilfegruppen“ als eigenständiger Fachdienst entwickelt (NAKOS) ${ }^{2}$ wo vor Ort für alle Interessierten (Betroffene wie Fachleute) themen-übergreifend Information und Beratung in Sachen Selbsthilfegruppen angeboten wird. Inzwischen gibt es ca. 300 solcher Einrichtungen in ganz Deutschland. $\mathrm{Zu}$ den grundlegenden Aufgaben dieser Selbsthilfe-Kontaktstellen gehört es,

- Interessenten - Betroffene wie professionelle Helfer - über das allgemeine Konzept von Selbsthilfegruppen zu informieren

- den Zugang zu bestehenden Gruppen zu eröffnen

- bei der Gründung neuer Gruppen behilflich zu sein

- bestehende Gruppen bei Problemen zu beraten

\footnotetext{
${ }^{2}$ Nationale Kontakt- und Informationsstelle zur Anregung und Unterstützung von Selbsthilfegruppen, Berlin
} 
- als „Drehscheibe“ den Selbsthilfegruppen-Bereich mit dem professionellen Versorgungssystem zu verknüpfen

- den Ansatz der eigenverantwortlichen Selbsthilfegruppen-Arbeit öffentlich zu vertreten.

\subsection{Modellprogramme}

Im Jahre 1987 wurde von der deutschen Bundesregierung ein Modellprogramm ins Leben gerufen, in dem an 18 Standorten die Arbeits- und Wirkungsweise solcher Kontaktstellen erprobt wurde. Die wissenschaftliche Begleitforschung ergab, grob gesagt, dass sich in Regionen mit Kontaktstellen mehr Selbsthilfegruppen bilden, dass sich ihnen mehr Menschen anschließen und dass sie stabiler arbeiten können. Kontaktstellen sind demnach der Königsweg einer Förderung des SelbsthilfegruppenEngagements der Bevölkerung durch eine unterstützende Infrastruktur und fachliche Beratung. Ein ganz ähnlich geartetes Modellprogramm in den neuen Bundesländern (der früheren Deutschen Demokratischen Republik) ab 1992 erbrachte im Grunde gleiche Resultate. Dies war so nicht unbedingt zu erwarten, da der dortigen Bevölkerung ja die Erfahrung mit selbst organisierter Gruppenarbeit außerhalb staatlicher Strukturen noch weitgehend fremd war.

\subsection{Gesetzgebung}

In einer Novellierung der deutschen Gesundheitsgesetzgebung zum 1. Januar 1993 tauchten erstmals die Begriffe „Selbsthilfegruppe“ und „Kontaktstelle“ in einem Gesetzestext auf (§ 20 SGB V). Es hieß dort, dass Krankenkassen ${ }^{3}$ diese fördern „können“ (!). Was politisch einen enormen Durchbruch zur gesellschaftlichen Anerkennung von Selbsthilfegruppen bedeutete, wurde von den Krankenkassen jedoch kaum genutzt. Ihre Förderung blieb insgesamt äußerst gering, zudem unsystematisch und intransparent. Hier wurde im Rahmen der Gesundheitsreform 2000 vom Gesetzgeber ein deutliches Signal gegeben: In der neuen Fassung jenes Paragraphen (§ 20, 4, SGB V) heißt es seit 1. Januar 2000 verpflichtend, dass die gesetzlichen Krankenkassen Selbsthilfegruppen, Selbsthilfeorganisationen und Selbsthilfekontaktstellen fördern "sollen“ (was in der Sprache der Juristen eine rechtlich bindende Formulierung darstellt) und dass Vertreter der Selbsthilfe an der Ausarbeitung von gemeinsamen Förderrichtlinien zu beteiligen sind. Insgesamt ist eine Summe von (damals) $1 \mathrm{DM}$, jetzt $0,56 €$ pro Versicherten und Jahr für die Selbsthilfe-Förderung bereitzustellen. Dies bedeutete eine erhebliche Ausweitung der Selbsthilfe-Förderung durch die Krankenkassen

\footnotetext{
${ }^{3}$ Das Gesundheitswesen in Deutschland wird nicht - wie z. B. in Großbritannien - aus Steuermitteln finanziert, sondern aus Beiträgen der sozialversicherungspflichtig beschäftigten Bevölkerung zu einer gesetzlichen Krankenversicherung, die sich in etwa 200 „Krankenkassen“ untergliedert. Krankenkassen sind also nicht staatlich, sondern „Körperschaften öffentlichen Rechts“; sie erwirtschaften keine Gewinne.
} 
und trug deutlich zur Stabilisierung der gesundheitsbezogenen Arbeit von Selbsthilfegruppen, Selbsthilfeorganisationen und Selbsthilfekontaktstellen bei. Leider erfolgte die praktische Umsetzung der Förderung vor Ort bzw. auf Landesebene nur äußerst schwerfällig. Im Jahre 2006 wurden gerade einmal 71\% der gesetzlich vorgeschriebenen Selbsthilfe-Förderung ausgegeben. Mit einer Neufassung (jetzt $\S 20 \mathrm{c}$ SGB V) versucht der Gesetzgeber seit 1. Januar 2008, hier Abhilfe zu schaffen. Seit 1. Juli 2001 ist eine analoge Formulierung im $\S 29$ SGB IX (,Rehabilitations-Gesetz“) in Kraft. Da es sich hierbei jedoch nicht um ein sog. Leistungsgesetz handelt, das Ansprüche auf bestimmte Leistungen oder finanzielle Unterstützung definieren würde, hat sich die Fördersituation der Selbsthilfe dadurch praktisch nicht geändert. Es stellt lediglich einen Appell an die Rehabilitationsträger (vor allem die Rentenversicherung) dar, Selbsthilfe auch finanziell zu fördern.

Eine große Gefahr wird von allen an diesen Reformen Beteiligten, und vor allem von der Selbsthilfe, darin gesehen, dass in dem erzwungenen deutlich stärkeren Einstieg der gesetzlichen Krankenversicherung in die Selbsthilfe-Förderung von der öffentlichen Hand (Städte, Landkreise, Bundesländer und die Bundesrepublik selber) ein willkommener Anlass gesehen werden könnte, das kommunale und staatliche Engagement zu reduzieren. Dies würde jedoch eine bloße Umverteilung der Lasten bedeuten: von Steuermitteln (die alle Bürger zu zahlen haben) hin zu Versicherungsbeiträgen (die nur gesetzlich Versicherte treffen, nicht aber besserverdienende Privatversicherte, Beamte usw.). Das darf nicht passieren, denn Selbsthilfe-Förderung ist und bleibt eine gesamtgesellschaftliche Gemeinschaftsaufgabe!

\section{Referenzen}

Borgetto, B. (2004) Selbsthilfe und Gesundheit: Analysen, Forschungsergebnisse und Perspektiven (Bern: Huber).

Borkman, TH. (1999) Understanding Self-Help / Mutual Aid: Experential Learning in the Commons (New Brunswick: Rutgers UP).

HÄRTER, M., A. LOH \& C. SPIES, Hrsg. (2005) „, Gemeinsam entscheiden - erfolgreich behandeln ": Neue Wege für Ärzte und Patienten (Köln: Deutscher Ärzte).

Herzog-Diem, R. \& S. Huber (2007) Selbsthilfe in Gruppen (Zürich: Beobachter).

HÖFLICH, A., F. MEYER, J. MATZAT \& M.E. BEUTEL (2007) Selbsthilfegruppen für psychisch und psychosomatisch Kranke: Zugangswege, Barrieren, Nutzen (Bremerhaven: Wirtschaftsverlag NW).

MAtzat, J. (2004) Wegweiser Selbsthilfegruppen: Eine Einführung für Laien und Fachleute (Gießen: Psychosozial).

MAtzAT, J. (in press-a) 'Ehrenamtliches Engagement, kollektive Selbsthilfe und politische Beteiligung im Gesundheitswesen' in TH. OlK, A. KleIN \& B. HARTnUss, Hrsg. (in press) Engagementpolitik: Die Entwicklung der Zivilgesellschaft als politische Aufgabe (Wiesbaden: Sozialwissenschaften).

MAtZAT, J. (in press-b) 'Selbsthilfegruppen als Beitrag zu einer psychosomatischen Medizin' in R. Adler, P. Joraschky, K. Köhle, W. Langwitz \& W. SÖllner, Hrsg. (in press) Uexküll Psychosomatische Medizin (7. Aufl.; München: Elsevier). 
Moeller, M.L. (1996) Selbsthilfegruppen: Anleitungen und Hintergründe (Reinbek: Rowohlt).

MOELLER, M.L. (2007) Anders helfen: Selbsthilfegruppen und Fachleute arbeiten zusammen (Gießen: Psychosozial).

Putnam, R. (2000) Bowling Alone: The Collapse and Revival of American Community (New York: Simon \& Schuster).

SCHMIDBAUER, W. (1977) Hilflose Helfer: Über die seelische Problematik der helfenden Berufe (Reinbek: Rowohlt).

SCHMIDBAUER, W. (2007) Das Helfersyndrom (Reinbek: Rowohlt).

Selbsthilfegruppenjahrbuch (1999 bis 2009) (Gießen: Deutsche Arbeitsgemeinschaft Selbsthilfegruppen e.V.).

SennetT, R. (1998) Der flexible Mensch: Die Kultur des neuen Kapitalismus (Berlin: Berlin).

SLESINA, W. \& A. KNERR (2007) Zusammenarbeit von Ärzten und Selbsthilfegruppen: Formen, Nutzen, Wünsche (Bremerhaven: Wirtschaftsverlag NW).

Sozialgesetzbuch (Berlin: Steffen Wasmund) heruntergeladen am 1. Dez. 2008 von http://www. sozialgesetzbuch-bundessozialhilfegesetz.de/.

TrojAn, A. \& A. EstorfF-KLEE, Hrsg. (2004) 25 Jahre Selbsthilfeunterstützung: Unterstützungserfahrungen und -bedarf am Beispiel Hamburgs (Münster: LIT). 